Yearbook of Population Research in Finland 30 (1992), pp 68-80

\title{
Long Range Population Prospects of Finland in the European Context
}

\author{
WOLFGANG LUTZ
}

Leader, Docent

Population Projects

IIASA

\section{CHRISTOPHER PRINZ}

Research Associate

IIASA

\section{Introduction}

Defining and calculating widely diverging alternative scenarios for the more distant future has become a favorite activity of scientists in the field of global and regional environmental change. In the absence of any certainty about trends in the coming decades, it turns out to be a useful exercise for understanding the phenomena and for finding appropriate policies for sustainable development to look at a number of alternative if-then relationships: If the $\mathrm{CO}_{2}$ concentration in the atmosphere doubled by 2030 then certain effects on the climate could be expected. Or in demography: If fertility and mortality remained at their present level, then the population size of Finland would decline to 3.6 million by the year 2050 and 2.3 million by the year 2100; if, alternatively, fertility increased to replacement level by 2000 , everything else remaining the same, then the Finnish population would increase slightly during the first half of the next century and then remain constant at about 5 million.

Any consistent set of assumptions about future trends may be called a scenario. As compared to scenarios on the above-mentioned environmental questions or any kind of economic forecasting, demographic scenarios have the great advantage that, given a starting population, assumptions on only three variables-namely fertility, mortality and migration-completely determine the size and age structure of the population at any point in the future. Other disciplines with many more uncertainties about the consequences of a given set of assumptions look with envy at demography. And yet demographers do not seem to enjoy this opportunity much, judging from the lack of alternative projections assuming demographic discontinuities.

Traditionally, population projection efforts concentrate on producing one most likely set of assumptions based on the continuation of present trends or sometimes based on the wishful thinking that fertility levels would increase to replacement level. Projections are usually presented in the form of a medium variant which is surrounded 
by a high and a low variant based on slightly different assumptions. This basic belief in structural stability is a pragmatic approach to short and medium term forecasts. For studying longer term population prospects and for considering the possibility of even short and medium term demographic discontinuities (for which an example is the postwar baby boom), a broader approach with a larger number of alternative scenarios is more appropriate. These scenarios should all be theoretically possible but need not be very likely according to our present thinking.

In formulating a broad range of possible alternative assumptions about future trends in fertility, mortality and migration, we need not take the extreme approach of viewing these trends as completely undetermined random processes. There is a large stock of knowledge in biology, medicine, sociology, psychology, economics and other disciplines that is relevant to our understanding about possible future changes in human longevity, reproduction and international migratory flows. If possible demographic discontinuities or surprises could be anticipated at all, this cannot be done by the analysis of past trends but by considering knowledge in this broader socioeconomic, cultural and biological context.

This interdisciplinary context is the focus of a recent book entitled Future Demographic Trends in Europe and North America: What Can We Assume Today? edited by W. Lutz (1991). In this book 31 authors give their views about possible future trends in mortality, fertility and international migration. Considerations on longevity in Part I range from a survey of currently used assumptions to the construction of a limit life table, the consideration of occupational mortality differentials and possible impacts of AIDS, adverse environmental factors, new biotechnologies and health promotion programs. Part II considers the future of reproduction: A survey of currently used assumptions, family size distributions, birth expectations, social and cultural factors associated with motherhood, relevant policies and the concept of stabilization after the fertility transition. Part III finally looks at migration from a social, economic and political point of view and considers several country case studies. In the final chapter, the alternative views expressed by the experts are translated into numbers which serve as input to a set of ten different scenario calculations for Western Europe, Eastern Europe, and North America.

This article will try to put the specific case of Finland into the context of the abovedescribed book. Based on the substantive articles in the book, scenarios for Finland will be defined in analogy to those assumed for Western Europe. Hence, in the following paper the logic behind the different scenario specifications will not be discussed extensively. For these questions we refer the reader to the abovementioned book. In terms of results we will compare the figures for Finland to those obtained for Eastern and Western Europe.

\section{Definition of scenarios}

Table 1 gives a full account of the assumptions which entered the different scenario calculations that were performed for Finland. The population starts in the year 1985 with the given age and sex composition of the Finnish population of that year. The population is grouped into 5-year age groups and the projection is performed in 5-year steps. For the calculations DIALOG was used, a user-friendly piece of software for multi-state population projections developed at IIASA (see Scherbov and Grechucha, 1988).

First a constant rates scenario (Scenario 0) assumes that all rates remain constant at their 1985-87 level. Next the assumptions made in the UN medium variant for 
Finland are taken as a point of reference (Scenario 1). All other scenarios (2 to 10) correspond directly to the scenario definitions made for Europe in Lutz (1991). Since the starting values for Finland in 1985-87 are quite similar to the average starting values in Western Europe in 1985, the path of change between the starting year and the assumed levels in the next century is also almost identical in the Finnish scenarios to those for Western Europe.

Next it is assumed that fertility levels increase to replacement level (i.e. a Total Fertility Rate (TFR) of 2.1) by the year 2000 (Scenario 2), assumptions on mortality stemming from the UN scenario. In contrast, the rapid fertility decline scenario (Scenario 3) assumes a decrease of the TFR to 1.46 in 2000 and 1.10 in 2025 . In the mortality stagnation scenario (Scenario 4) no further improvements in life expectancy

Table 1. Assumptions of 11 demographic scenarios for Finland.

Scenario

0 Constant Rate

1 UN Medium Variant

2 Replacement Fertility

3 Fertility Decline

4 Mortality Stagnation

5 Mortality Decline

6 Fertility and mortality Decline

Moderate migration

8 High migration

9 Scenario $3+8$ combined

10 Scenario $6+8$ combined

\section{Fertility \\ (TFR)}

1.60

1.60

1.60

1.65

1.70

1.80

1.65

2.10

2.10

1.60

1.46

1.10

1.65

1.70

1.80

1.65

1.70

1.80

1.60

1.46

1.10

1.65

1.70

1.80

1.65

1.70

1.80

1.60

1.46

1.10

1.60

1.46

1.10
Mortality (Life expectancy)

Women Men

$\begin{array}{ll}78.7 & 70.7\end{array}$

$\begin{array}{ll}78.7 & 70.7\end{array}$

$\begin{array}{ll}78.7 & 70.7\end{array}$

$78.8 \quad 71.0$

$80.2 \quad 72.9$

$83.0 \quad 76.2$

$\begin{array}{ll}78.8 & 71.0\end{array}$

$80.2 \quad 72.9$

$83.0 \quad 76.2$

$\begin{array}{ll}78.8 & 71.0\end{array}$

$80.2 \quad 72.9$

$83.0 \quad 76.2$

$\begin{array}{ll}78.7 & 70.7\end{array}$

$\begin{array}{ll}78.7 & 70.7\end{array}$

$\begin{array}{ll}78.7 & 70.7\end{array}$

$\begin{array}{ll}78.8 & 71.0\end{array}$

$83.4 \quad 76.4$

$95.0 \quad 90.0$

$78.8 \quad 71.0$

$83.4 \quad 76.4$

$95.0 \quad 90.0$

$78.8 \quad 71.0$

$80.2 \quad 72.9$

$83.0 \quad 76.2$

78.8

80.2

83.0

78.8

80.2

83.0

78.8

83.4

95.0
Net migration Per year

\section{0}

0

0

0

0

0

0

0

0

0

0

0

0

0

0

0

0

0

0

0

0

$+10000$

$+10000$

$+10000$

$71.0+30000$

$72.0+30000$

$76.2+30000$

$71.0+30000$

$72.9+30000$

$76.2+30000$

$71.0+30000$

$76.4+30000$

$90.0+30000$

Starting year of projections is 1985; observed fertility and mortality between 1985 and 1987 ; linear interpolation between the intervals given above; after 2025 fertility, mortality and migration are kept constant. 
are assumed. The mortality decline scenario (Scenario 5), on the other hand, assumes further increases in life expectancy at birth resulting in 90 years for men and 95 years for women after the year 2025. The fertility and mortality decline scenario (Scenario 6 ) is a combination of Scenarios 3 and 5. Finally, the moderate migration scenario (Scenario 7) assumes an annual net inflow of 10,000 foreigners into Finland each year, whereas the high migration scenario (Scenario 8) assumes a net gain of 30,000 persons per year. The age and sex composition of the migrants was assumed to be identical to those in the European scenarios, namely $52 \%$ men and $48 \%$ women with a mean age of around 25 years. The last two scenarios considered are combinations of fertility decline and high immigration (Scenario 9) and of fertility decline, mortality decline and high immigration (Scenario 10).

\section{Resulting changes in population size}

Table 2a gives the total population size of Finland, the corresponding annual number of births and the average annual natural growth rates of the population for all scenarios considered. Data are given in ten-year intervals up to 2030, then for 2050, and even for 2100 to demonstrate the very long term impact of the rates considered in the various scenarios.

Concerning the total population size over the next 40 years (i.e. by 2030), a range is opened from between 4.36 million in the case of all rates remaining constant and 6.16 million in the case of the high immigration scenario. In the latter case about 1.2 million of the population will not be born in Finland. By the year 2050 the range is further opened with the fertility decline scenario resulting in 3.49 million at the lower end and again the high immigration scenario with 6.6 million at the top. By the year 2100 these two extreme scenarious would result in 1.36 versus 7.79 million people living in Finland (see Figure 1).

The absolute number of births per year under the various scenarios mirrors the patterns described above. Under the UN scenario (Scenario 1) the number of births declines from 63,000 in 1985 to 43,000 by the year 2050 . Under the high immigration scenario, however, the number of births would increase to 74,000 in 2050 . A comparison of these two figures shows that, in the high immigration scenario, almost half of the births in 2050 would be of other than Finnish ethnic origin. Under the replacement fertility scenario, the number of births would stabilize slightly above 60,000 per year whereas in the fertility decline scenario it would be as low as 17,000 in the year 2050 .

A look at the average annual rates of natural growth (which is determined by the balance of deaths and births excluding migration) shows that, in the medium term, only a few scenarios result in negative growth rates, whereas in the longer term all of them show negative growth rates. The reason for this lies in the present age structure of the Finnish population, which still has relatively large cohorts in the reproductive ages. In the coming decades, however, the aging of the population will be so strong that even an increase of fertility to replacement level cannot counterbalance the large number of deaths due to the large number of old people.

Finland has presently $1.3 \%$ of the population of the total Western European population of 384 million. The population of Eastern Europe including the European republics of the Soviet Union (and the Asian part of the Russian Republic) stands presently around 355 million. Table $2 \mathrm{~b}$ shows that the scenarios for Eastern and Western Europe open up a range of possible future population sizes similar to that discussed above for Finland. The proportion of the Finnish population in all of West- 
Table 2a. Population size and growth, Finland 1990-2100.

a. Total population size (in 100)

\begin{tabular}{|c|c|c|c|c|c|c|c|}
\hline & & 1990 & 2000 & 2010 & 2020 & 2030 & 2050 \\
\hline 0 & Constant Rate & 4971 & 4968 & 4862 & 4671 & 4365 & 3663 \\
\hline 1 & UN medium & & 5013 & 4995 & 4929 & 4777 & 4293 \\
\hline 2 & Repl.Fertility & & 5013 & 5124 & 5166 & 5140 & 5019 \\
\hline 3 & Fert.Decline & & 5013 & 4941 & 4754 & 4422 & 3492 \\
\hline 4 & Mort.Stagnation & & 4988 & 4910 & 4764 & 4523 & 3977 \\
\hline 5 & Mort.Decline & & 5013 & 5036 & 5119 & 5172 & 4973 \\
\hline 6 & Fer./Mor.Decline & & 5013 & 4981 & 4944 & 4816 & 4166 \\
\hline 7 & Mod. Migration & & 5185 & 5302 & 5383 & 5391 & 5231 \\
\hline 8 & High Migration & & 5250 & 5531 & 5868 & 6163 & 6637 \\
\hline 9 & Sce. $3+8$ combined & & 5250 & 5469 & 5656 & 5705 & 5502 \\
\hline & Sce. $6+8$ combined & & 5250 & 5511 & 5854 & 6121 & 6261 \\
\hline
\end{tabular}

b. Number of births (in 1000)

\begin{tabular}{|c|c|c|c|c|c|c|c|}
\hline & & 1990 & 2000 & 2010 & 2020 & 2030 & 2050 \\
\hline 0 & Constant Rate & 61 & 51 & 49 & 44 & 39 & 33 \\
\hline 1 & UN medium & & 54 & 53 & 50 & 47 & 43 \\
\hline 2 & Repl.Fertility & & 67 & 65 & 60 & 63 & 62 \\
\hline 3 & Fert.Decline & & 50 & 42 & 34 & 27 & 17 \\
\hline 4 & Mort.Stagnation & & 54 & 53 & 50 & 46 & 42 \\
\hline 5 & Mort.Decline & & 54 & 53 & 50 & 47 & 43 \\
\hline 6 & Fer./Mor.Decline & & 50 & 42 & 34 & 27 & 18 \\
\hline 7 & Mod. Migration & & 57 & 58 & 57 & 55 & 55 \\
\hline 8 & High Migration & & 58 & 62 & 66 & 68 & 74 \\
\hline 9 & Sce. $3+8$ combined & & 54 & 50 & 44 & 39 & 34 \\
\hline 10 & Sce. $6+8$ combined & & 54 & 50 & 44 & 39 & 34 \\
\hline
\end{tabular}

c. Average annual natural growth rate of the population (in \%)

\begin{tabular}{|c|c|c|c|c|c|c|c|c|}
\hline & & 1990 & 2000 & 2010 & 2020 & 2030 & 2050 & 2100 \\
\hline 0 & Constant Rate & $0.2 \%$ & $-0.1 \%$ & $-0.3 \%$ & $-0.6 \%$ & $-0.8 \%$ & $-0.9 \%$ & $-1.0 \%$ \\
\hline 1 & UN medium & & $0.0 \%$ & $-0.0 \%$ & $-0.2 \%$ & $-0.5 \%$ & $-0.6 \%$ & $-0.5 \%$ \\
\hline 2 & Repl.Fertility & & $0.3 \%$ & $0.2 \%$ & $0.0 \%$ & $-0.1 \%$ & $-0.1 \%$ & $0.0 \%$ \\
\hline 3 & Fert.Decline & & $-0.0 \%$ & $-0.3 \%$ & $-0.5 \%$ & $-0.9 \%$ & $-1.4 \%$ & $-2.2 \%$ \\
\hline 4 & Mort.Stagnation & & $-0.1 \%$ & $-0.2 \%$ & $-0.4 \%$ & $-0.7 \%$ & $-0.6 \%$ & $-0.6 \%$ \\
\hline 5 & Mort.Decline & & $0.0 \%$ & $0.2 \%$ & $0.2 \%$ & $-0.0 \%$ & $-0.3 \%$ & -0.5 \\
\hline 6 & Fer./Mor.Decline & & $-0.0 \%$ & $0.0 \%$ & $-0.1 \%$ & $-0.4 \%$ & $-1.0 \%$ & $-1.9 \%$ \\
\hline 7 & Mod. Migration & & $0.1 \%$ & $0.0 \%$ & $-0.1 \%$ & $-0.3 \%$ & $-0.4 \%$ & $-0.3 \%$ \\
\hline 8 & High Migration & & $0.1 \%$ & $0.1 \%$ & $0.1 \%$ & $-0.1 \%$ & $-0.1 \%$ & -0.1 \\
\hline 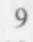 & Sce. $3+8$ combined & & $0.0 \%$ & $-0.1 \%$ & $-0.3 \%$ & $-0.6 \%$ & $-0.9 \%$ & -1.1 \\
\hline 0 & Sce. $6+8$ combined & & $0.0 \%$ & $0.1 \%$ & $0.1 \%$ & $-0.2 \%$ & $-0.5 \%$ & -0.9 \\
\hline
\end{tabular}

Table 2b. Population size, Europe 1990-2050.

Total population size (in millions)

\begin{tabular}{|c|c|c|c|c|}
\hline & \multicolumn{3}{|c|}{ Western Europe } \\
\hline & & 1990 & 2020 & 205 \\
\hline 1 & UN medium & 384 & 392 & \\
\hline 2 & Repl.Fertility & & 415 & \\
\hline 3 & Fert.Decline & & 367 & \\
\hline 4 & Mort.Stagnation & & 382 & \\
\hline 5 & Mort.Decline & & 416 & \\
\hline 6 & Fer./Mor.Decline & & 390 & \\
\hline 7 & Mod. Migration & & 426 & \\
\hline 8 & High Migration & & 448 & \\
\hline 9 & Sce. $3+8$ combined & & 420 & \\
\hline 10 & Sce. $6+8$ combined & & 444 & \\
\hline
\end{tabular}

Eastern Europe

$\begin{array}{rrr}1990 & 2020 & 2050 \\ 355 & 386 & 378 \\ & 401 & 422 \\ & 367 & 289 \\ & 372 & 348 \\ & 411 & 440 \\ & 391 & 348 \\ & 376 & 371 \\ & 387 & 434 \\ & 368 & 339 \\ & 392 & 398\end{array}$


Figure 1. Total population size in Finland, 1990-2100 by scenario.
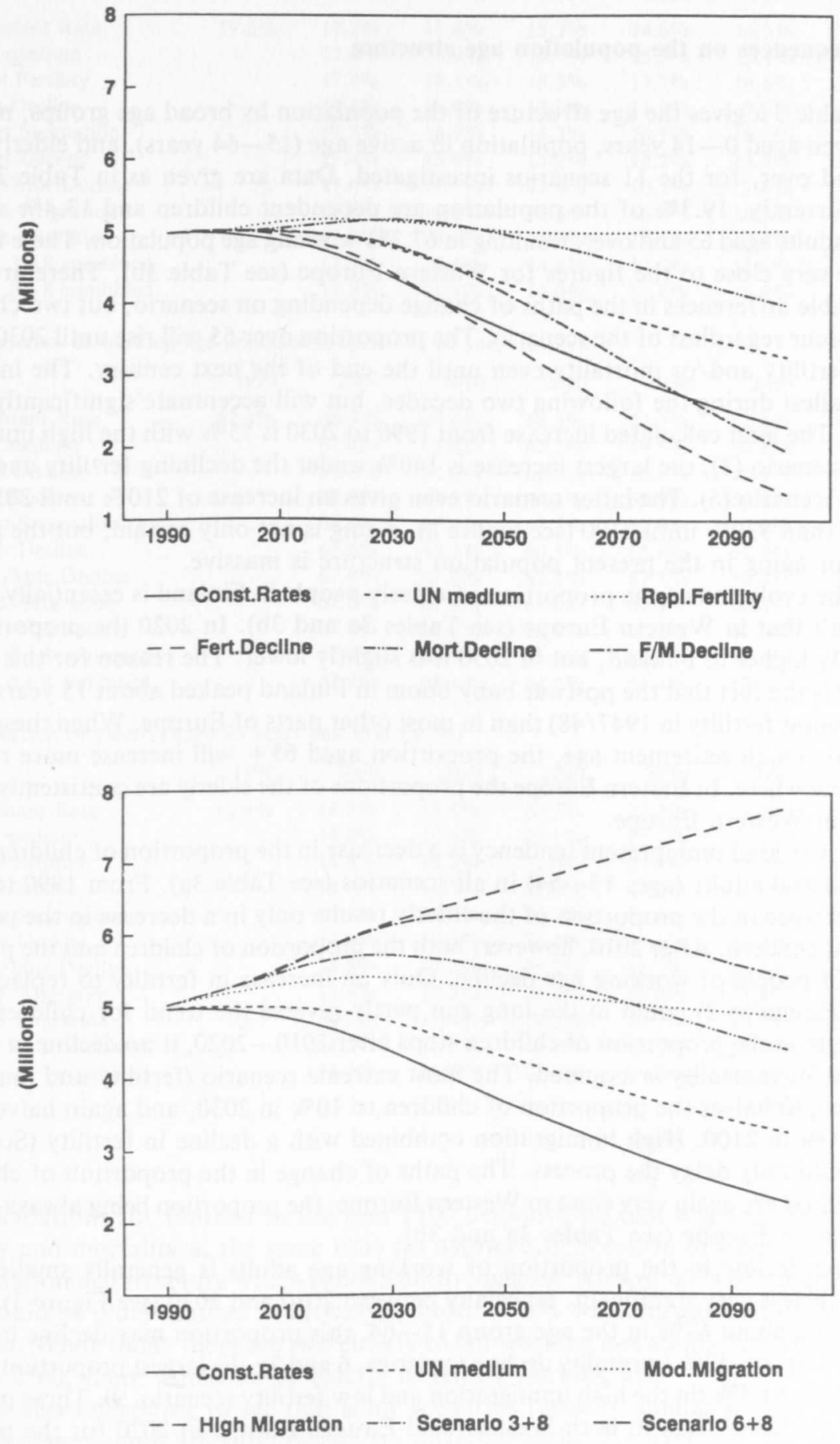
ern Europe is unlikely to change under any given scenario. Only if demographic trends in Finland evolved differently from those in the rest of Europe will the proportion of Finns in Europe change.

\section{Consequences on the population age structure}

Table 3 a gives the age structure of the population by broad age groups, namely children aged $0-14$ years, population in active age (15-64 years), and elderly aged 65 and over, for the 11 scenarios investigated. Data are given as in Table $2 \mathrm{a}$.

Currently, $19.3 \%$ of the population are dependent children and $13.4 \%$ dependent adults aged 65 and over, resulting in $67.3 \%$ working age population. These figures come very close to the figures for Western Europe (see Table $3 \mathrm{~b}$ ). There are considerable differences in the paths of change depending on scenario, but two changes will occur regardless of the scenario. The proportion over 65 will rise until 2030, with low fertility and/or mortality even until the end of the next century. The increase is smallest during the following two decades, but will accentuate significantly after 2010. The least calculated increase from 1990 to 2030 is $55 \%$ with the high immigration scenario (8), the largest increase is $140 \%$ under the declining fertility and mortality scenario (6). The latter scenario even gives an increase of $210 \%$ until 2050 and more than $330 \%$ until 2100 (see Figure 2). Aging is not only certain, but the potential for aging in the present population structure is massive.

The evolution of the proportion of elderly people in Finland is essentially parallel with that in Western Europe (see Tables 3a and 3b). In 2020 the proportion is slightly higher in Finland, but in 2050 it is slightly lower. The reason for this divergence is the fact that the postwar baby boom in Finland peaked about 15 years earlier (highest fertility in 1947/48) than in most other parts of Europe. When these large cohorts reach retirement age, the proportion aged $65+$ will increase more rapidly than elsewhere. In Eastern Europe the proportions of the elderly are consistently lower than in Western Europe.

The second omnipresent tendency is a decrease in the proportion of children (ages 0-14) and adults (ages 15-64) in all scenarios (see Table 3a). From 1990 to 2010 the increase in the proportion of the elderly results only in a decrease in the proportion of children. After 2010, however, both the proportion of children and the proportion of people of working age decline. Only an increase in fertility to replacement level (Scenario 2) could in the long run partly reverse the trend for children. The decrease in the proportion of children stops after 2010-2020, if no decline in fertility and/or mortality is assumed. The most extreme scenario (fertility and mortality decline, 6) halves the proportion of children to $10 \%$ in 2030 , and again halves it to only $5 \%$ in 2100 . High immigration combined with a decline in fertility (Scenario 9) would only delay the process. The paths of change in the proportion of children in Finland are again very close to Western Europe, the proportion being always higher in Eastern Europe (see Tables $3 a$ and $3 b$ ).

The decline in the proportion of working age adults is generally smaller, but nevertheless very significant, especially between 2010 and 2030 (see Figure 3). With presently about $67 \%$ in the age group $15-64$, this proportion may decline by 2050 to around $50-52 \%$ (mortality decline scenarios, 6 and 7); the largest proportion found in 2050 is $64.4 \%$ (in the high immigration and low fertility scenario, 9). These proportions are lower than in both Western and Eastern Europe in 2020 for the reasons described above, and somewhere in between the two regions in 2050 (see Tables $3 \mathrm{a}$ and $3 \mathrm{~b})$. 
Table 3a. Population age structure, Finland 1990-2100.

a. Proportion of children, aged $0-14$ years (in \%)

\begin{tabular}{|c|c|c|c|c|c|c|c|}
\hline & 1990 & 2000 & 2010 & 2020 & 2030 & 2050 & 2100 \\
\hline 0 Constant Rate & $19.3 \%$ & $17.2 \%$ & $15.6 \%$ & $15.3 \%$ & $14.6 \%$ & $14.5 \%$ & $14.4 \%$ \\
\hline $1 \mathrm{UN}$ medium & & $17.4 \%$ & $15.9 \%$ & $15.7 \%$ & $15.3 \%$ & $15.4 \%$ & $15.5 \%$ \\
\hline 2 Repl.Fertility & & $17.4 \%$ & $18.1 \%$ & $18.3 \%$ & $17.7 \%$ & $18.8 \%$ & $19.0 \%$ \\
\hline 3 Fert.Decline & & $17.4 \%$ & $15.0 \%$ & $13.0 \%$ & $11.0 \%$ & $9.0 \%$ & $7.4 \%$ \\
\hline 4 Mort.Stagnation & & $17.5 \%$ & $16.2 \%$ & $16.3 \%$ & $16.1 \%$ & $16.5 \%$ & $16.7 \%$ \\
\hline Mort.Decline & & $17.4 \%$ & $15.8 \%$ & $15.2 \%$ & $14.2 \%$ & $13.4 \%$ & $13.0 \%$ \\
\hline 6 Fer./Mor.Decline & & $17.4 \%$ & $14.9 \%$ & $12.6 \%$ & $10.1 \%$ & $7.6 \%$ & $5.2 \%$ \\
\hline 7 Mod. Migration & & $17.8 \%$ & $16.5 \%$ & $16.4 \%$ & $16.1 \%$ & $16.2 \%$ & $16.4 \%$ \\
\hline High Migration & & $17.9 \%$ & $17.0 \%$ & $17.2 \%$ & $17.1 \%$ & $17.3 \%$ & $17.1 \%$ \\
\hline Sce. $3+8$ combined & & $17.9 \%$ & $16.0 \%$ & $14.4 \%$ & $12.7 \%$ & $11.1 \%$ & $10.7 \%$ \\
\hline e. $6+8$ combined & & $17.9 \%$ & $15.9 \%$ & $14.0 \%$ & $11.8 \%$ & & $8.8 \%$ \\
\hline
\end{tabular}

b. Proportion of working age population, aged 15-64 years (in \%)

\begin{tabular}{|c|c|c|c|c|c|c|c|c|}
\hline & & 1990 & 2000 & 2010 & 2020 & 2030 & 2050 & 2100 \\
\hline 0 & Constant Rate & $67.3 \%$ & $68.3 \%$ & $68.6 \%$ & $64.3 \%$ & $62.7 \%$ & $62.4 \%$ & $62.3 \%$ \\
\hline 1 & UN medium & & $67.8 \%$ & $67.4 \%$ & $62.2 \%$ & $59.5 \%$ & $58.8 \%$ & $59.3 \%$ \\
\hline 2 & Repl.Fertility & & $67.8 \%$ & $65.7 \%$ & $60.6 \%$ & $58.9 \%$ & $59.1 \%$ & $60.4 \%$ \\
\hline 3 & Fert.Decline & & $67.8 \%$ & $68.2 \%$ & $64.1 \%$ & $61.9 \%$ & $59.2 \%$ & $51.4 \%$ \\
\hline 4 & Mort.Stagnation & & $68.0 \%$ & $68.2 \%$ & $63.7 \%$ & $62.0 \%$ & $62.2 \%$ & $62.9 \%$ \\
\hline 5 & Mort.Decline & & $67.8 \%$ & $67.1 \%$ & $60.5 \%$ & $55.8 \%$ & $51.8 \%$ & $50.1 \%$ \\
\hline 6 & Fer./Mor.Decline & & $67.8 \%$ & $67.8 \%$ & $62.2 \%$ & $57.7 \%$ & $50.8 \%$ & $36.9 \%$ \\
\hline 7 & Mod. Migration & & $67.8 \%$ & $67.6 \%$ & $63.0 \%$ & $60.9 \%$ & $60.4 \%$ & $61.0 \%$ \\
\hline $0^{\circ}$ & High Migration & & $67.9 \%$ & $67.7 \%$ & $63.6 \%$ & $62.2 \%$ & $62.5 \%$ & $62.4 \%$ \\
\hline 9 & Sce. $3+8$ combined & & $67.9 \%$ & $68.5 \%$ & $65.7 \%$ & $65.0 \%$ & $64.4 \%$ & $61.3 \%$ \\
\hline 10 & Sce. $6+8$ combined & & $67.9 \%$ & $68.1 \%$ & $64.0 \%$ & $61.4 \%$ & $57.7 \%$ & $51.3 \%$ \\
\hline
\end{tabular}

c. Proportion of elderly, aged 65 years and over (in \%)

\begin{tabular}{|c|c|c|c|c|c|c|c|c|}
\hline & & 1990 & 2000 & 2010 & 2020 & 2030 & 2050 & 2100 \\
\hline 0 & Constant Rate & $13.4 \%$ & $14.5 \%$ & $15.8 \%$ & $20.5 \%$ & $22.7 \%$ & $23.1 \%$ & $23.4 \%$ \\
\hline 1 & UN medium & & $14.8 \%$ & $16.6 \%$ & $22.1 \%$ & $25.1 \%$ & $25.9 \%$ & $25.2 \%$ \\
\hline 2 & Repl.Fertility & & $14.8 \%$ & $16.2 \%$ & $21.0 \%$ & $23.4 \%$ & $22.1 \%$ & $20.6 \%$ \\
\hline 3 & Fert.Decline & & $14.8 \%$ & $16.8 \%$ & $22.9 \%$ & $27.2 \%$ & $31.8 \%$ & $41.3 \%$ \\
\hline 4 & Mort.Stagnation & & $14.5 \%$ & $15.7 \%$ & $20.1 \%$ & $21.9 \%$ & $21.3 \%$ & $20.4 \%$ \\
\hline 5 & Mort.Decline & & $14.8 \%$ & $17.1 \%$ & $24.4 \%$ & $30.0 \%$ & $34.8 \%$ & $36.9 \%$ \\
\hline 6 & Fer./Mor.Decline & & $14.8 \%$ & $17.3 \%$ & $25.2 \%$ & $32.2 \%$ & $41.6 \%$ & $57.9 \%$ \\
\hline 7 & Mod. Migration & & $14.4 \%$ & $15.9 \%$ & $20.6 \%$ & $23.0 \%$ & $23.4 \%$ & $22.7 \%$ \\
\hline 8 & High Migration & & $14.2 \%$ & $15.4 \%$ & $19.2 \%$ & $20.7 \%$ & $20.3 \%$ & $20.5 \%$ \\
\hline 9 & Sce. $3+8$ combined & & $14.2 \%$ & $15.5 \%$ & $19.9 \%$ & $22.3 \%$ & $24.5 \%$ & $28.0 \%$ \\
\hline 10 & Sce. $6+8$ combined & & $14.2 \%$ & $16.0 \%$ & $22.0 \%$ & $26.7 \%$ & $32.4 \%$ & $39.9 \%$ \\
\hline
\end{tabular}

Calculations for Finland to the year 2100 demonstrate that a strong decline in fertility and mortality at the same time (as assumed in Scenario 6) would result in a population age structure which is difficult to imagine: Almost $60 \%$ of the population would be older than 65 in 2100 , only around $35 \%$ working age adults and $5 \%$ children. While today there are two elderly to ten working age adults, under Scenario 6 in 2100 , there would be 15 elderly to ten of working age.

It is also interesting to see that some scenarios result in similar proportions in the major age groups after following very different paths. For example, the replacement fertility scenario (2) and the fertility decline scenario (3) lead to the same proportion of working age adults in 2050 , namely $59 \%$. The proportion of children, how- 
ever, is twice as high in Scenario 2 than in Scenario 3. The proportion of the elderly differs in the opposite direction. It also happens that different assumptions lead to a similar age structure but very different total population sizes. Scenarios 4 (mortality stagnation) and 8 (high immigration), for instance, show almost identical proportions at different ages in the long run, but total population size is only 3 million in 2100 under Scenario 4 while it is almost 7.8 million under Scenario 8 (see Tables $2 a$ and $3 a$ ).

If one is looking for a single summary indicator describing population aging, then the mean age of the population provides a useful measure. Table 4 gives those mean ages of the population under the different scenarios for Finland and for Europe.

Table 3b. Population age structure, Europe 1990-2050.

a. Proportion of children, aged $0-14$ years (in \%)

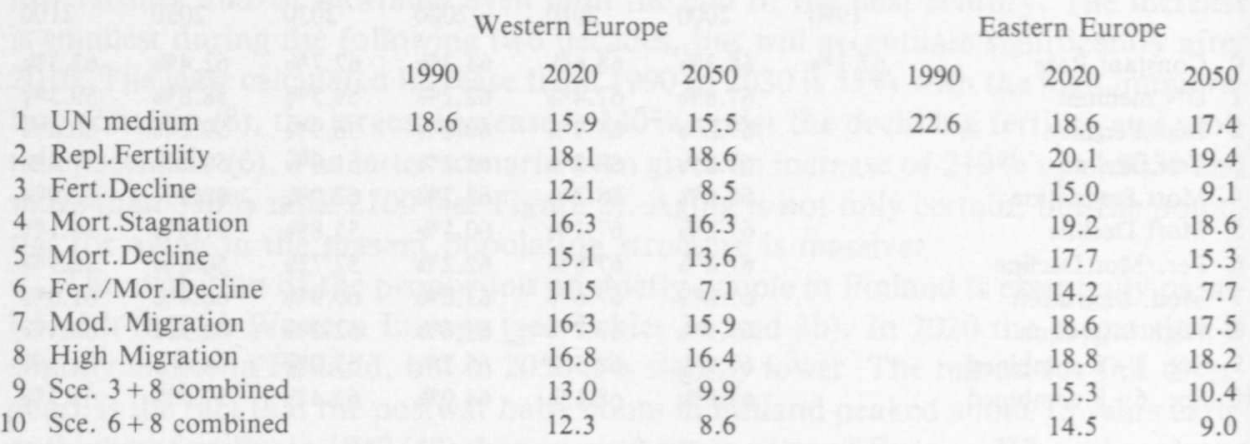

b. Proportion of working age population, aged $15-64$ years (in \%)

Western Europe

Eastern Europe

$\begin{array}{llllll}1990 & 2020 & 2050 & 1990 & 2020 & 2050 \\ 67.3 & 63.6 & 58.4 & 66.9 & 63.8 & 60.3 \\ & 62.6 & 59.4 & & 63.0 & 60.6 \\ & 65.9 & 57.2 & & 66.4 & 61.8 \\ & 64.7 & 61.1 & & 65.3 & 63.6 \\ & 60.7 & 51.5 & & 61.1 & 53.7 \\ & 62.8 & 48.7 & & 63.5 & 53.1 \\ & 64.1 & 59.4 & & 63.8 & 60.4 \\ & 64.6 & 61.0 & & 64.0 & 62.1 \\ & 67.1 & 61.4 & & 66.7 & 64.4 \\ & 64.2 & 53.9 & & 63.8 & 56.6\end{array}$

c. Proportion of elderly, aged 65 years and over (in \%)

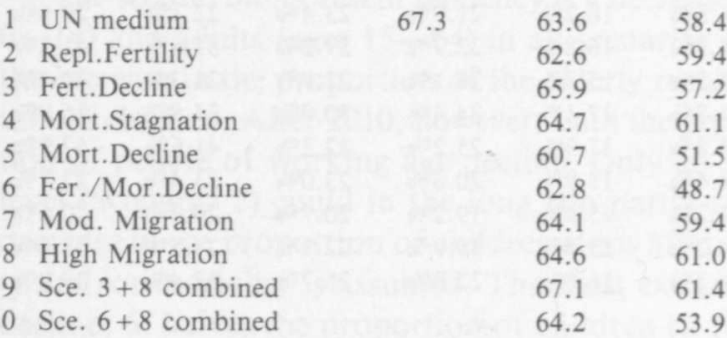

Western Europe

Eastern Europe

$\begin{array}{lll}1990 & 2020 & 2050 \\ 14.2 & 20.5 & 26.1 \\ & 19.4 & 22.1 \\ & 22.0 & 34.3 \\ & 19.0 & 22.6 \\ & 24.2 & 35.0 \\ & 25.8 & 44.2 \\ 19.6 & 24.7 \\ & 18.7 & 22.6 \\ 19.9 & 28.8 \\ & 23.5 & 37.5\end{array}$

$\begin{array}{rrr}1990 & 2020 & 2050 \\ 10.5 & 17.6 & 22.2 \\ & 17.0 & 19.9 \\ & 18.6 & 29.1 \\ & 15.5 & 17.8 \\ & 21.3 & 31.0 \\ & 22.3 & 39.2 \\ 17.6 & 22.2 \\ & 17.2 & 19.7 \\ 18.1 & 25.2 \\ & 21.8 & 34.4\end{array}$


Figure 2. Proportion of the elderly (aged $65+$ ), Finland, 1990-2100 by scenario.

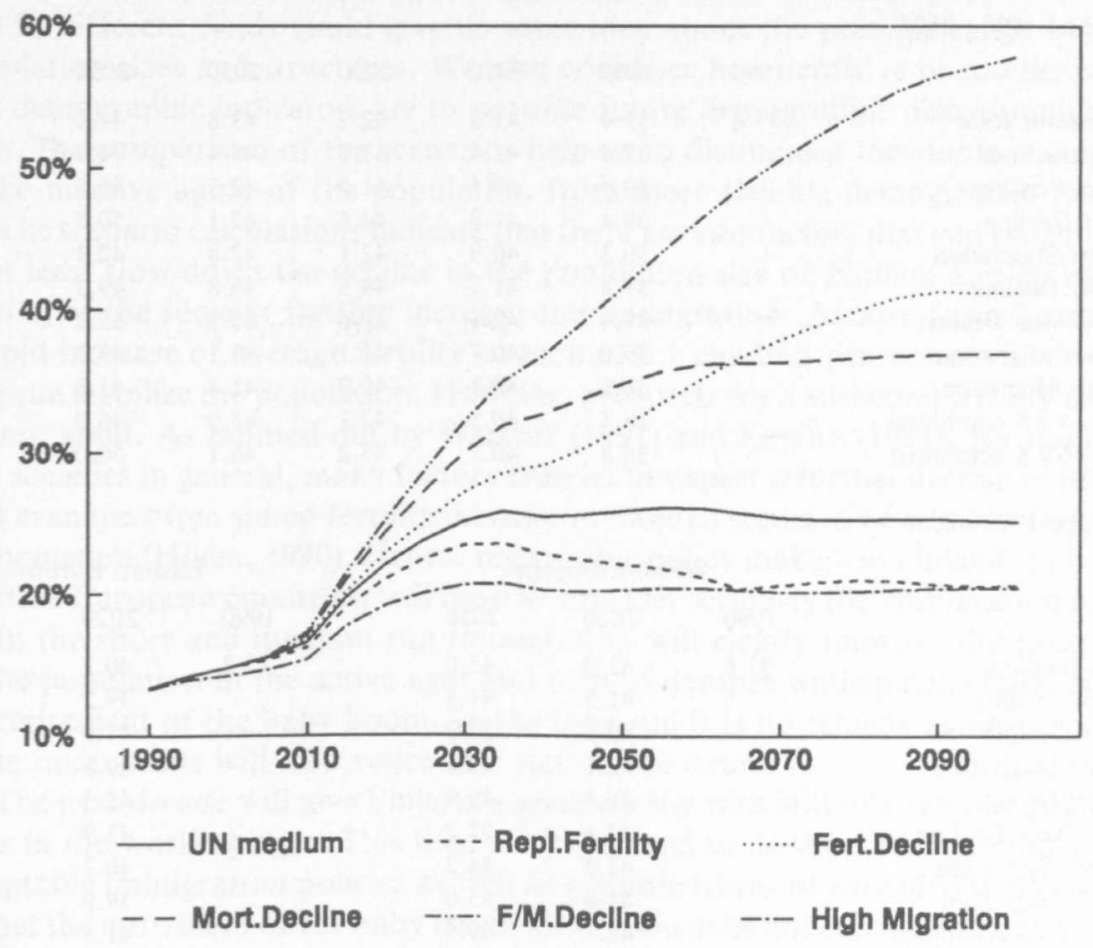

Figure 3. Proportion of the active (aged 15-64), Finland, $1990-2100$ by scenario.

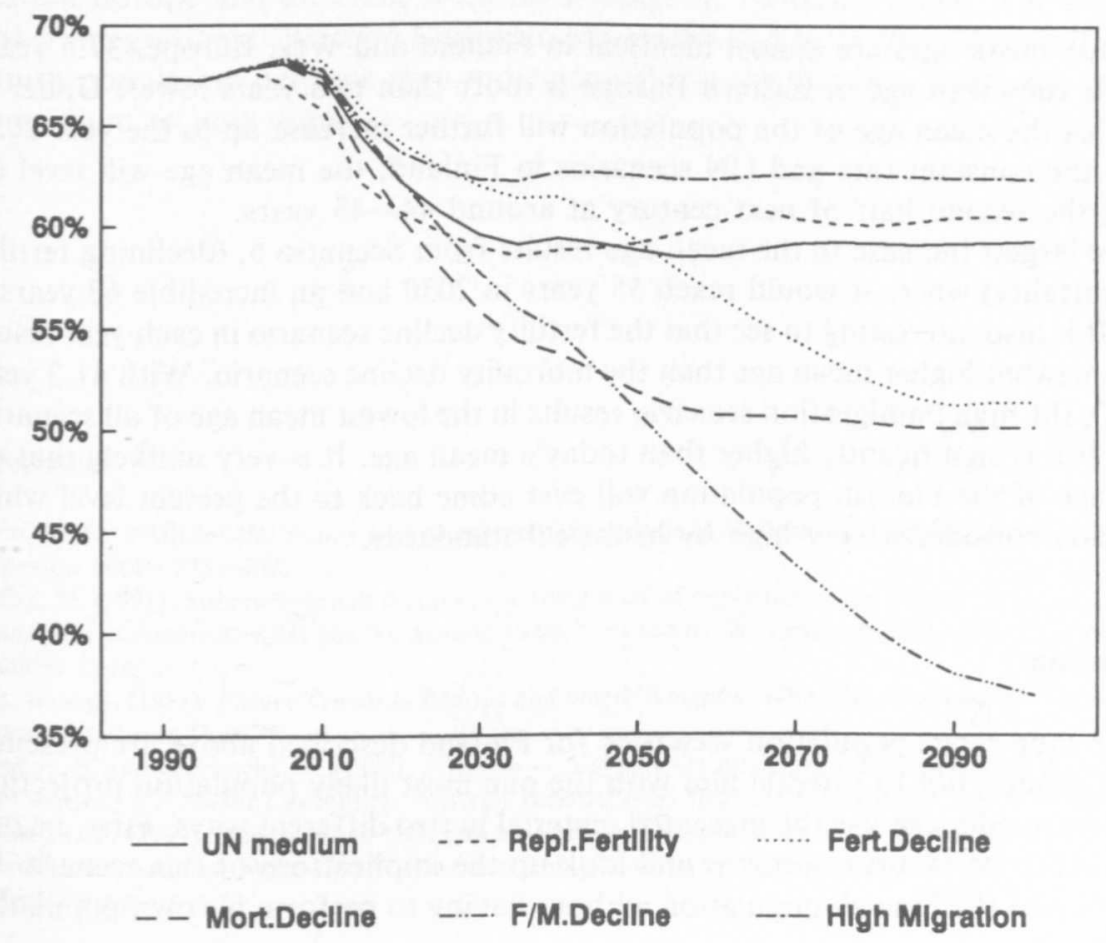


Table 4. Mean age of the population (in years).

a. Finland $1990-2100$

\begin{tabular}{|c|c|c|c|c|c|c|c|}
\hline & 1990 & 2000 & 2010 & 2020 & 2030 & 2050 & 2100 \\
\hline 0 Constant Rate & 37.4 & 39.4 & 41.2 & 42.7 & 43.8 & 44.2 & 44.3 \\
\hline 1 UN medium & & 39.4 & 41.4 & 43.1 & 44.4 & 44.7 & 44.4 \\
\hline 2 Repl.Fertility & & 39.4 & 40.5 & 41.6 & 42.3 & 41.5 & 40.9 \\
\hline 3 Fert.Decline & & 39.4 & 41.8 & 44.4 & 47.1 & 50.7 & 55.0 \\
\hline 4 Mort.Stagnation & & 39.3 & 40.9 & 42.1 & 42.8 & 42.4 & 42.0 \\
\hline 5 Mort.Decline & & 39.4 & 41.7 & 44.3 & 46.8 & 49.3 & 50.4 \\
\hline 6 Fer./Mor.Decline & & 39.4 & 42.1 & 45.6 & 49.5 & 55.2 & 62.7 \\
\hline 7 Mod. Migration & & 39.0 & 40.7 & 42.1 & 43.1 & 43.3 & 42.8 \\
\hline 8 High Migration & & 38.8 & 40.1 & 40.9 & 41.4 & 41.3 & 41.5 \\
\hline 9 Sce. $3+8$ combined & & 38.8 & 40.5 & 42.1 & 43.9 & 46.2 & 47.6 \\
\hline 10 Sce. $6+8$ combined & & 38.8 & 40.7 & 43.2 & 46.1 & 50.0 & 53. \\
\hline
\end{tabular}

b. Europe 1990-2050

\begin{tabular}{|c|c|c|c|c|c|c|}
\hline & \multicolumn{3}{|c|}{ Western Europe } & \multicolumn{3}{|c|}{ Eastern Europe } \\
\hline & 1990 & 2020 & 2050 & 1990 & 2020 & 2050 \\
\hline $1 \mathrm{UN}$ medium & 37.6 & 42.9 & 45.0 & 35.2 & 40.2 & 42.5 \\
\hline 2 Repl.Fertility & & 41.3 & 41.8 & & 39.2 & 40.6 \\
\hline 3 Fert.Decline & & 45.1 & 52.2 & & 41.9 & 49.8 \\
\hline 4 Mort.Stagnation & & 42.1 & 43.1 & & 39.1 & 40.0 \\
\hline 5 Mort.Decline & & 44.8 & 49.9 & & 42.3 & 47.4 \\
\hline 6 Fer./Mor.Decline & & 47.1 & 57.2 & & 43.9 & 54.9 \\
\hline 7 Mod. Migration & & 42.3 & 44.2 & & 40.2 & 42.4 \\
\hline 8 High Migration & & 41.6 & 43.0 & & 39.9 & 40.9 \\
\hline 9 Sce. $3+8$ combined & & 43.7 & 49.1 & & 41.5 & 47.3 \\
\hline 10 Sce. $6+8$ combined & & 45.5 & 53.5 & & 43.5 & 52.0 \\
\hline
\end{tabular}

Presently mean ages are almost identical in Finland and West Europe, 37.5 years, whereas the mean age in Eastern Europe is more than two years lower. Under all scenarios the mean age of the population will further increase up to the year 2030 . Under the constant rate and UN scenarios in Finland, the mean age will level off during the second half of next century at around $44-45$ years.

The largest increase in the mean age results from Scenario 6, (declining fertility and mortality) where it would reach 55 years in 2030 and an incredible 63 years in 2100. It is also interesting to see that the fertility decline scenario in each year results in a somewhat higher mean age than the mortality decline scenario. With 41.3 years in 2050 , the high immigration scenario results in the lowest mean age of all scenarios which still is significantly higher than today's mean age. It is very unlikely that the mean age of the Finnish population will ever come back to the present level which is already considered very high by historical standards.

\section{Conclusion}

The long range population scenarios for Finland described above are presented for the reader, not to provide him with the one most likely population projection, but so the reader can use the presented material in two different ways. First, he may choose his own favorite scenario and look up the implications of this scenario for the future of the Finnish population without having to perform his own population 
projections. Second, and more importantly, this set of widely diverging scenarios based on assumptions reflecting the opposing opinions of a large number of scientists in different fields could give us some idea about the possible range of future population sizes and structures. We also could see how sensitive or insensitive certain demographic indicators are to possible future demographic discontinuities. Finally, the comparison of the scenarios help us to distinguish inevitable trends such as the massive aging of the population from more flexible demographic features.

The scenario calculations indicate that there are two factors that can possibly stop or at least slow down the decline in the population size of Finland and an extreme greying of the society: fertility increase and immigration. As seen from Scenario 3, a rapid increase of average fertility to around 2.1 children per woman could in the long run stabilize the population. However, prospects for a sustained fertility increase are not good. As pointed out by Westoff (1991) and Keyfitz (1991), for industrialized societies in general, many factors even let us expect a further decline in fertility. And even the often stated fertility increase in Sweden seems to be a rather short term phenomenon (Hoem, 1990). Hence, responsible policy makers in Finland, as in other Western European countries, will have to consider seriously the immigration option.

In the short and medium run immigration will clearly improve the proportion of the population in the active ages and help to dampen anticipated crises, such as the retirement of the baby boom. In the long run it is no remedy against aging because immigrants will also retire and stay in the country.

The next decade will give Finland a grace period with still relatively large proportions in the working ages. This time could be used to develop rational and socially acceptable immigration policies as well as possible forms of extended working ages, so that the retirement of the baby boom generation does not catch the country unprepared.

Finally, the comparison of Finnish population patterns to Eastern and Western Europe shows that, in demographic terms, Finland is very close to the average of Western Europe and different from the average of Eastern Europe. The expected further integration of Western European economies and societies is likely to link the Finnish population patterns even more strongly to the Western European ones in the medium to long term.

\section{References}

Hoem, J.M. (1990). Social policy and recent fertility change in Sweden. Population and Development Review 16(4): 735-748.

Keyfitz, N. (1991). Subreplacement fertility: the third level of explanation. In: Future Trends in Europe and North America: what can we assume today?, edited by W. Lutz, pp. 235-246. London: Academic Press.

Lutz, W. (ed). (1991). Future Trends in Europe and North America: What Can We Assume Today? London: Academic Press.

Scherbov, S. and Grechucha, V. (1988). »DIAL» - A system for modeling multidimensional demographic processes. WP-88-36. Laxenburg, Austria: International Institute for Applied Systems Analysis.

Westoff, C.F. (1991). The return to replacement fertility: a magnetic force. In: Future Trends in Europe and North America: What Can We Assume Today?, edited by W. Lutz, pp. 227-233. London: Academic Press. 


\section{Abstract}

The population of Finland is projected along eleven different scenarios assuming widely diverging alternative trends in fertility, mortality and migration up to the year 2100. The definitions of these scenarios follow those of a recent study (Lutz, 1991) on Europe and North America. They range from constant rates to assuming replacement fertility versus a Total Fertility Rate (TFR) of 1.1, mortality stagnation versus a strong increase in life expectancy, and no immigration versus 30,000 migrants per year. The results show that no matter what scenario is chosen, the next 30 years will bring an enormous increase of the population over age 65 . The proportion in working age will be relatively stable up to the year 2010 and then strongly decline under all conditions, which is a consequence of the Finnish baby boom of the late 1940s. Projected total population sizes in 2050 will range from 3.5 million in the fertility decline scenario to 6.6 million in the high immigration scenario. 